\title{
Plasma Vanin-1 as a Novel Biomarker of Sepsis for Trauma Patients: A Prospective Multicenter Cohort Study
}

\author{
Hongxiang Lu $\cdot$ Anqiang Zhang $\cdot$ Dalin Wen $\cdot$ Juan Du \\ Jianhui Sun · Liang Qiao · Dingyuan Du · Wei Gu (1) · \\ Jianxin Jiang
}

Received: December 17, 2020 / Accepted: February 4, 2021 / Published online: February 23, 2021

(c) The Author(s) 2021

\section{ABSTRACT}

Introduction: Vanin-1 plays a pivotal role in oxidative stress and the inflammatory response. However, its relationship with traumatic sepsis remains unknown. The aim of our study was to evaluate whether plasma vanin-1 could be used for the early prediction of traumatic sepsis.

Methods: In this three-stage prospective cohort study, severe trauma patients admitted from January 2015 to October 2018 at two hospitals

Supplementary Information The online version contains supplementary material available at https:// doi.org/10.1007/s40121-021-00414-w.

H. Lu

Department of Traumatic Orthopaedics, General Hospital of Xinjiang Military Region, Ürümqi, China

W. Gu ( $₫)$

School of Medicine, Chongqing University, Chongqing, China

e-mail: clgwjm@163.com

H. Lu - A. Zhang · D. Wen · J. Du · J. Sun · L. Qiao . J. Jiang $(\bowtie)$

State Key Laboratory of Trauma, Burns and Combined Injury, Wound Trauma Medical Center, Institute of Surgery Research, Daping Hospital, Army Medical University, Chongqing, China e-mail: hellojjx@126.com

D. Wen · D. Du

Chongqing Emergency Medical Center, Chongqing University Central Hospital, School of Medicine, Chongqing University, Chongqing, China were enrolled. Plasma vanin-1 levels were measured by enzyme-linked immunosorbent assay (ELISA). The associations among variables and traumatic sepsis were identified by logistic regression models and the receiver operating characteristic (ROC) curve was analyzed to evaluate the diagnostic efficiency.

Results: A total of 426 trauma patients (22 in the discovery cohort, 283 in the internal test cohort, and 121 in the external validation cohort) and 16 healthy volunteers were recruited. The plasma vanin-1 of trauma patients was significantly higher than that of healthy volunteers $(P<0.05)$. Patients with sepsis had higher plasma vanin-1 than patients without sepsis in the discovery trauma cohort $(P<0.05)$. In the internal test cohort, plasma vanin-1 at day 1 after trauma was significantly associated with the incidence of sepsis $(\mathrm{OR}=3.92$, $95 \%$ CI $2.68-5.72, P=1.62 \times 10^{-12}$ ). As a predictive biomarker, vanin-1 afforded a better area under the curve (AUC) $(0.82,95 \%$ CI $0.77-0.87)$ than C-reaction protein (CRP) $(0.62,95 \%$ CI 0.56-0.68, $P<0.0001$ ), procalcitonin (PCT) $(0.66,95 \%$ CI $0.60-0.71, P<0.0001)$, and Acute Physiology and Chronic Health Evaluation II (APACHE II) $(0.71,95 \%$ CI $0.65-0.76, P=6.70$ $\left.\times 10^{-3}\right)$. The relevance was further validated in the external validation cohort $(\mathrm{OR}=4.26,95 \%$ CI 2.22-8.17, $P=1.28 \times 10^{-5}$ ), with an AUC of 0.83 (95\% CI 0.75-0.89). Vanin-1 could also improve the diagnostic efficiency of APACHE II $(\mathrm{AUC}=0.85)$. 
Conclusions: Our study demonstrated that plasma vanin-1 increased among trauma patients and was independently associated with the risk of sepsis. Vanin-1 might be a potential biomarker for the early prediction of traumatic sepsis.

Trial Registration: Clinicaltrials.gov Identifier, NCT01713205.

Keywords: Biomarker; Sepsis; Trauma; Vanin-1

\begin{tabular}{|c|c|}
\hline \multicolumn{2}{|c|}{ Abbreviations } \\
\hline APACHE II & $\begin{array}{l}\text { Acute Physiology and Chronic } \\
\text { Health Evaluation II }\end{array}$ \\
\hline AUC & Area under the curve \\
\hline CRP & C-reaction protein \\
\hline ELISA & $\begin{array}{l}\text { Enzyme-linked immunosorbent } \\
\text { assay }\end{array}$ \\
\hline ISS & Injury Severity Score \\
\hline LPS & Lipopolysaccharides \\
\hline PCT & Procalcitonin \\
\hline $\operatorname{PPAR} \gamma$ & $\begin{array}{l}\text { Peroxisome proliferator-activated } \\
\text { receptor- } \gamma\end{array}$ \\
\hline ROC & $\begin{array}{l}\text { Receiver operating characteristic } \\
\text { curve }\end{array}$ \\
\hline SD & Standard deviation \\
\hline SOFA & $\begin{array}{l}\text { Sequential Organ } \\
\text { Assessment }\end{array}$ \\
\hline VNN1 & Vanin-1 \\
\hline
\end{tabular}

\section{Key Summary Points}

Sepsis has become the main cause of inhospital death in severe trauma patients. New predictive clinical biomarkers with high specificity are urgently needed.

Vanin-1 was reported to play a pivotal role in oxidative stress and the inflammatory response. We hypothesized that vanin-1 might act as a potential predictive biomarker of traumatic sepsis.

Plasma vanin-1 increased among trauma patients and was independently associated with the risk of sepsis, especially in the first 3 days after injury.
There is a significant relationship between plasma vanin- 1 and sepsis in both the internal test cohort and the external validation cohort. These results contribute to the body of evidence supporting the use of plasma vanin- 1 in the early prediction of traumatic sepsis.

Further studies with multiple populations and functional experiments are needed.

\section{DIGITAL FEATURES}

This article is published with digital features, including a summary slide, to facilitate understanding of the article. To view digital features for this article go to https://doi.org/10.6084/ m9.figshare.13691686.

\section{INTRODUCTION}

Sepsis is one of the most common complications and the leading cause of in-hospital death for severe trauma patients $[1,2]$. Sepsis results in a longer length of hospital stay and higher health care costs, which greatly increases the burden on society $[3,4]$. However, the diagnosis of post-traumatic sepsis is notoriously difficult in the sense that trauma patients are in a state of "sterile inflammation" [5]. The strong oxidative stress induced by severe injury may produce a genomic storm with alteration of up to $80 \%$ of the leukocyte transcriptome, thereby largely affecting the parameters used to diagnose sepsis [6]. Considering the consequences of sepsis after trauma, early recognition and individualized therapy of those patients at high risk of sepsis are essential prerequisites to reduce the morbidity of trauma patients $[7,8]$. Therefore, an ideal biomarker is required to facilitate the early prediction of post-injury sepsis.

Vanin-1 (vascular non-inflammatory molecule 1, VNN1) is a pantetheinase that hydrolyzes pantetheine to pantothenic acid (vitamin $\mathrm{B}_{5}$ ) and cysteamine. Beyond its function in coenzyme A (CoA) metabolism, VNN1 
has been found to participate in both oxidative stress and the inflammatory response $[9,10]$. $V N N 1^{-l-}$ mice showed increased tolerance to oxidative stress caused by gamma-radiation or paraquat intoxication [11]. In addition, they showed an attenuated inflammatory reaction after infection by schistosomiasis [12] or rickettsiosis [13]. In patients with multiple injuries, physical damage is the initiating factor in the production of oxygen free radicals and inflammatory reactions, and numerous studies have demonstrated associations between injury and elevated oxidative stress and inflammation [14]. Therefore, we hypothesized that vanin-1 might increase in response to physiological stress in trauma patients and might act as a potential predictive biomarker of traumatic sepsis.

In the present study, we detected the change of plasma vanin-1 levels in trauma patients. Then, we investigated the relationship between vanin-1 expression and incidence of sepsis. In addition, a prospective cohort study was conducted to evaluate the predictive power of plasma vanin-1 for traumatic sepsis. Finally, an independent cohort was conducted to validate these findings. Our aim is to determine whether plasma vanin-1 could act as a potential biomarker for the early diagnosis of sepsis in trauma patients.

\section{METHODS}

\section{Study Design and Setting}

The present study was a two-center, prospective cohort investigation. Severe trauma patients with an Injury Severity Score (ISS) greater than 16 points and admitted to the hospital within $24 \mathrm{~h}$ post injury were enrolled in parallel from the ICU at the Department of Trauma Surgery from Daping Hospital and the Central Hospital of Chongqing University from January 2015 to October 2018. Patients who met one of the following criteria were excluded: (1) age less than 15 years old or more than 65 years old; (2) pregnancy; (3) malignant tumor; (4) serious chronic history of cardiovascular, respiratory, renal, hepatic, hematologic, or immunological diseases. Sixteen healthy volunteers were asymptomatic nonsmokers under 65 years of age, who had no known chronic medical conditions.

This study included a discovery cohort and two test cohorts. Patients were followed up during the hospital stay and divided into sepsis and non-sepsis groups during data analysis according to the occurrence of sepsis, which was defined as a suspected infection with an acute change in SOFA scores of at least 2 (Sepsis3) [15]. For those patients with sepsis enrolled before 2016 and first diagnosed on the basis of Sepsis-2, we invited clinical doctors to reanalyze whether the patients met the definition of Sepsis-3. The definition of infection was a clinically obvious source or positive bacterial culture. The research was approved by the Institutional Ethics Review Board of the Army Medical University. The study was performed in accordance with the Helsinki Declaration of 1964, and its later amendments. Informed consent of all patients was obtained from the patients or their relatives. The National Clinical Trial number is NCT01713205, registered on 22 October 2012.

\section{Data Collection}

Demographic characteristics and clinical data, including general condition, vital signs, and consciousness state, were retrieved from the electronic medical records. Acute Physiology and Chronic Health Evaluation (APACHE) II scores and Sequential Organ Failure Assessment (SOFA) scores were calculated to evaluate the disease severity and organ failures.

\section{Detection of Vanin-1 in Plasma}

Blood specimens were collected and processed as previously reported [2, 16]. Briefly, whole blood was collected using an EDTA-coated tube immediately after admission and kept at $4{ }^{\circ} \mathrm{C}$. Sample was centrifuged within $1 \mathrm{~h}$ at $3000 \mathrm{rpm}$ for $5 \mathrm{~min}$ at $4{ }^{\circ} \mathrm{C}$. The plasma was separated and stored at $-80^{\circ} \mathrm{C}$ for further analyses. To test the dynamic change in plasma vanin-1, blood samples at days $1,3,5,7$, and 14 after injury were collected in the discovery cohort. The 
basic levels of plasma vanin-1 were measured among 16 healthy volunteers. A commercially available enzyme-linked immunosorbent assay (ELISA) (Cloud-Clone Corp, USA) was conducted to detect vanin-1 in plasma. Vanin-1 levels were determined in duplicate following the manufacturer's instructions.

\section{Statistical Analysis}

Categorical variables were summarized as number and proportion, and the differences were compared with the $\chi^{2}$ test. Continuous variables were expressed as the median and standard deviation (SD), and comparisons of the mean were performed using Student's $t$ test or Mann-Whitney $U$ test. The associations among variables and traumatic sepsis were identified by logistic regression models. Additionally, we adjusted for age, sex, and ISS to correct the associations. The evaluation of the predictive accuracy was performed using the area under the curve (AUC) of the receiver operating characteristic (ROC) curve. The correlations between vanin-1 and other variables were assessed using Spearman rank correlation coefficient. Differences with $P<0.05$ were statistically significant. All statistical analyses were performed using SPSS version 17.0 (SPSS Inc., Chicago, USA) and MedCalc version 13 (MedCalc Software, Ostend, Belgium).

\section{RESULTS}

\section{Clinical Data and General Information}

In total, 426 patients suffering from severe injury were enrolled in our study, including 305 patients (11 patients with sepsis and 11 patients without sepsis were randomly selected as the discovery cohort, and the remaining 283 patients as the internal test cohort) from Daping Hospital, and 121 patients from Chongqing Emergency Medical Center as the external validation cohort. The age, gender, injuries, and infections among the three groups were comparable (Table 1). Most patients developed sepsis 3-5 days after injury. Gram-negative bacteria were the main pathogenic microorganisms. Just a few patients with sepsis tested positive for Gram-positive bacteria or had mixed infection. Pneumonia and primary bloodstream infections were the major infection sites. The mortality of both cohorts was relatively low, 3.18\% and $2.48 \%$, respectively.

\section{Dynamic Change of Plasma Vanin-1 After Injury}

The mean plasma vanin-1 levels were 0.66 (0.61-0.70) $\mathrm{ng} / \mathrm{ml}$ for healthy population. Trauma patients had the highest plasma vanin1 on the first day of admission $(1.73 \pm 1.07 \mathrm{ng} /$ $\mathrm{ml}, P=1.21 \times 10^{-4}$, discovery cohort) (Fig. 1a). Then, the level of vanin-1 gradually declined from day 3 to day 14 after injury but was still significantly higher than that of healthy controls $(P<0.01$ for all days). We further compared plasma levels of vanin-1 between the sepsis and the non-sepsis group. Significantly elevated plasma levels of vanin-1 were found in patients with sepsis, especially on early days after admission $(P=0.03$ for day 1 and $P=0.04$ for day 3 ), indicating the potentially predictive value of vanin-1 levels for sepsis in trauma patients (Fig. 1b). No significant difference was demonstrated between two groups at days 5, 7 , and 14 .

\section{Predictive Value of Plasma Vanin-1 in Trauma Patients}

To investigate whether plasma vanin-1 could be used as a predictive biomarker of traumatic sepsis, we evaluated the predictive power of plasma vanin- 1 at day 1 in 283 trauma patients (internal test cohort). Our results showed a strong association between higher plasma vanin-1 levels taken $24 \mathrm{~h}$ post injury and the incidence of secondary sepsis within the subsequent 14 days $\left(P=6.02 \times 10^{-13}\right)$ (Fig. $\left.2 \mathrm{a}\right)$. No significant correlation between ISS and plasma vanin-1 was observed $\left(r^{2}=0.01, P=0.06\right)$. In addition, our results demonstrated that the level of plasma vanin-1 at day 1 was significantly associated with the incidence of sepsis in patients after trauma $(\mathrm{OR}=3.89$, 
Table 1 Clinical characteristics of trauma patients

\begin{tabular}{|c|c|c|c|c|}
\hline Variables & $\begin{array}{l}\text { Discovery cohort } \\
(n=22)\end{array}$ & $\begin{array}{l}\text { Internal test cohort } \\
(n=283)\end{array}$ & $\begin{array}{l}\text { External validation cohort } \\
(n=121)\end{array}$ & $P$ value* \\
\hline Gender (female/male) & $4 / 18$ & $59 / 224$ & $28 / 93$ & 0.90 \\
\hline Age (years) & $44.75 \pm 15.02$ & $43.39 \pm 12.01$ & $44.48 \pm 12.65$ & 0.68 \\
\hline ISS & $26.85 \pm 9.19$ & $24.45 \pm 8.44$ & $22.97 \pm 8.56$ & 0.10 \\
\hline $\mathrm{AIS}_{\text {Head/neck }}$ & $2.55 \pm 1.76$ & $1.80 \pm 1.67$ & $1.80 \pm 1.65$ & 0.10 \\
\hline AIS $_{\text {Face }}$ & $0.40 \pm 0.60$ & $0.43 \pm 0.79$ & $0.28 \pm 0.59$ & 0.12 \\
\hline $\mathrm{AIS}_{\text {Thorax }}$ & $3.05 \pm 1.23$ & $2.71 \pm 1.26$ & $3.01 \pm 1.06$ & 0.43 \\
\hline $\mathrm{AIS}_{\text {Abdomen }}$ & $1.65 \pm 1.66$ & $1.26 \pm 1.52$ & $1.30 \pm 1.32$ & 0.47 \\
\hline $\mathrm{AIS}_{\text {Upper/lower extremity }}$ & $1.70 \pm 1.13$ & $2.17 \pm 1.49$ & $1.95 \pm 1.63$ & 0.07 \\
\hline GCS initial & $13.85 \pm 6.16$ & $14.00 \pm 3.00$ & $13.78 \pm 2.68$ & 0.32 \\
\hline APACHE II scores initial & $7.85 \pm 3.76$ & $7.67 \pm 6.31$ & $7.40 \pm 4.57$ & 0.92 \\
\hline SOFA scores initial & $1.85 \pm 1.18$ & $2.95 \pm 2.22$ & $2.28 \pm 1.41$ & 0.10 \\
\hline Sepsis, $n(\%)$ & - & $91(32.16 \%)$ & $41(33.88 \%)$ & 0.73 \\
\hline Pathogens, $n(\%)$ & & & & 0.46 \\
\hline Gram-negative & - & $62(68.13 \%)$ & $21(51.22 \%)$ & \\
\hline Gram-positive & - & $11(12.09 \%)$ & $8(19.51 \%)$ & \\
\hline $\begin{array}{l}\text { Mixed Gram-negative and } \\
\text { Gram-positive }\end{array}$ & - & $7(7.69 \%)$ & $5(12.20 \%)$ & \\
\hline Others & - & $4(4.40 \%)$ & $2(4.87 \%)$ & \\
\hline Negative blood cultures & - & $7(7.69 \%)$ & $5(12.20 \%)$ & \\
\hline Source of infection, $n(\%)$ & & & & 0.41 \\
\hline Blood & - & $25(27.47 \%)$ & $10(24.39 \%)$ & \\
\hline Sputum & - & $27(29.68 \%)$ & $13(31.71 \%)$ & \\
\hline Urine & - & $15(16.48 \%)$ & $8(19.51 \%)$ & \\
\hline Secretions & - & $18(19.78 \%)$ & $4(9.76 \%)$ & \\
\hline Others & - & $6(6.59 \%)$ & $6(14.63 \%)$ & \\
\hline Time trauma-sepsis onset, days & $5.85 \pm 2.91$ & $5.76 \pm 4.82$ & $5.03 \pm 2.52$ & 0.72 \\
\hline ICU days & $6.16 \pm 9.22$ & $4.47 \pm 7.92$ & $5.76 \pm 13.01$ & 0.99 \\
\hline Deaths & $0(0.00 \%)$ & $9(3.18 \%)$ & $3(2.48 \%)$ & 0.66 \\
\hline
\end{tabular}

ISS Injury Severity Score, AIS Abbreviated Injury Scale, GCS Glasgow Coma Scale, APACHE II Acute Physiology and Chronic Health Evaluation II, SOFA Sequential Organ Failure Assessment

${ }^{*}$ Categorical variables were compared using the $\chi^{2}$ test and continuous variables were compared using analysis of variance (ANOVA) test 
A

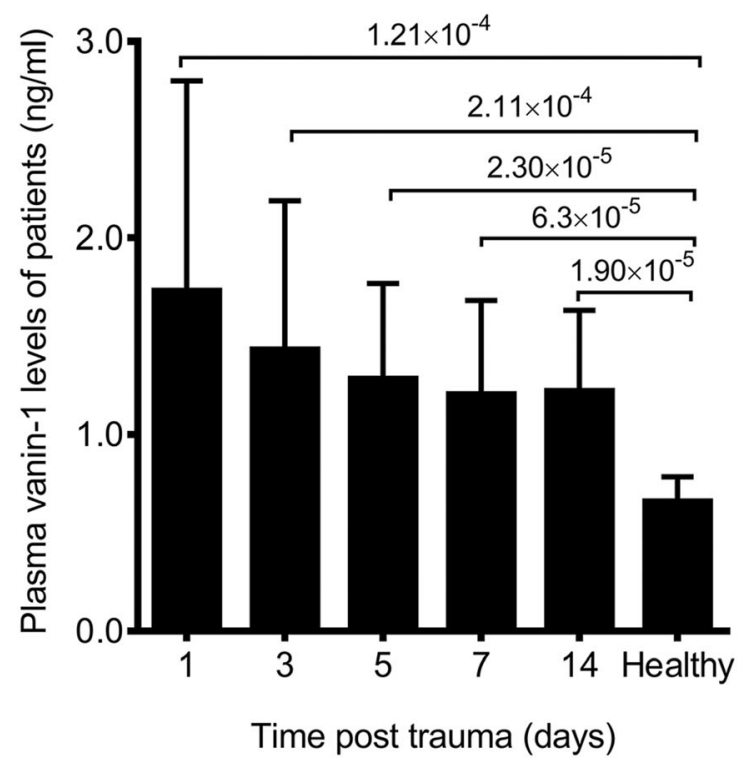

Fig. 1 Kinetics of plasma vanin-1 levels in trauma patients on admission and at days 3, 5, 7, and 14 during hospitalization from the discovery cohort. a Plasma vanin-1 of trauma patients is significantly higher than that of healthy volunteers $(n=22$ trauma patients vs. 16 healthy controls). b Trauma patients with sepsis have

95\% CI 2.68-5.63, $P=6.99 \times 10^{-13}$ ) (Table S1 in the supplementary material). Logistic regression analyses also demonstrated that plasma vanin-1 was significantly related to a higher occurrence of sepsis adjusted for age, sex, smoking, drinking, and ISS ( $\mathrm{OR}=3.92,95 \%$ CI $2.68-5.72, P=1.67 \times 10^{-12}$, Table 2). On the basis of the ROC analysis of plasma vanin-1 at day 1, an AUC of 0.82 (95\% CI 0.77-0.87) was obtained for the morbidity of sepsis after trauma (Fig. 3a). The optimal cutoff value was $1.41 \mathrm{ng} / \mathrm{ml}$ in the internal test cohort with the sensitivity and specificity of $70.00 \%$ and $84.90 \%$, respectively (Table S2 in the supplementary material). Although CRP, PCT, and APACHE II at day 1 after trauma were also associated with the risk of traumatic sepsis in our internal test cohort (Tables S1 and S2 in the supplementary material, and Table 2), our results revealed that vanin-1 had a better AUC than other biomarkers (CRP, $P<0.0001$; PCT,
B

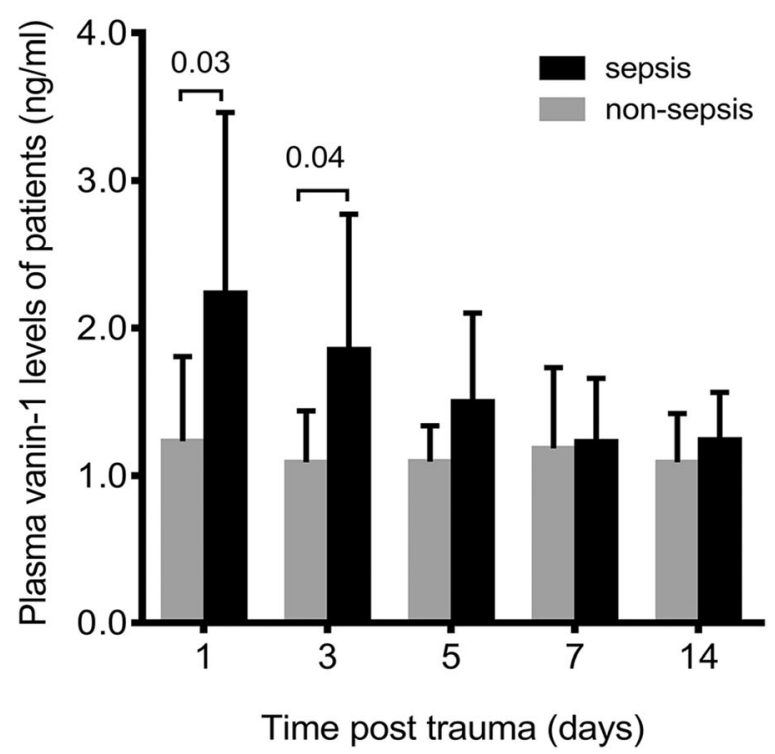

higher plasma vanin-1 than patients without sepsis at the early stage after injury $(n=11$ patients with sepsis vs. 11 patients without sepsis, $P=0.03$ for day 1 and $P=0.04$ for day 3). Data are expressed as the mean and 95\% CI. Statistical analysis comprised Student's $t$ test

$P<0.0001 ; \quad$ APACHE II, $\quad P=6.70 \times 10^{-3}$ ) (Fig. 3a).

\section{Validation of Vanin-1 for Predict Sepsis in Trauma Patients}

We further validated the predictive ability of plasma vanin-1 at day 1 after injury in the external validation cohort. In this cohort, plasma vanin-1 was also not associated with ISS $\left(r^{2}=0.03, P=0.07\right)$. Patients with sepsis had significant higher plasma vanin-1 than patients without sepsis at day 1 after injury $(P=8.39 \times$ $10^{-6}$ ) (Fig. 2b). Plasma vanin-1 at day 1 after injury was also associated with the risk of sepsis, adjusted for age, sex, smoking, drinking, and ISS $\left(\mathrm{OR}=4.26,95 \%\right.$ CI 2.22-8.17, $P=1.28 \times 10^{-5}$, Table 2). Plasma vanin-1 afforded an AUC of 0.83 (95\% CI 0.75-0.89) for the incidence of sepsis after trauma (Fig. 3b). The optimal cutoff value was $1.35 \mathrm{ng} / \mathrm{ml}$ in the external validation cohort, with a sensitivity and specificity of 


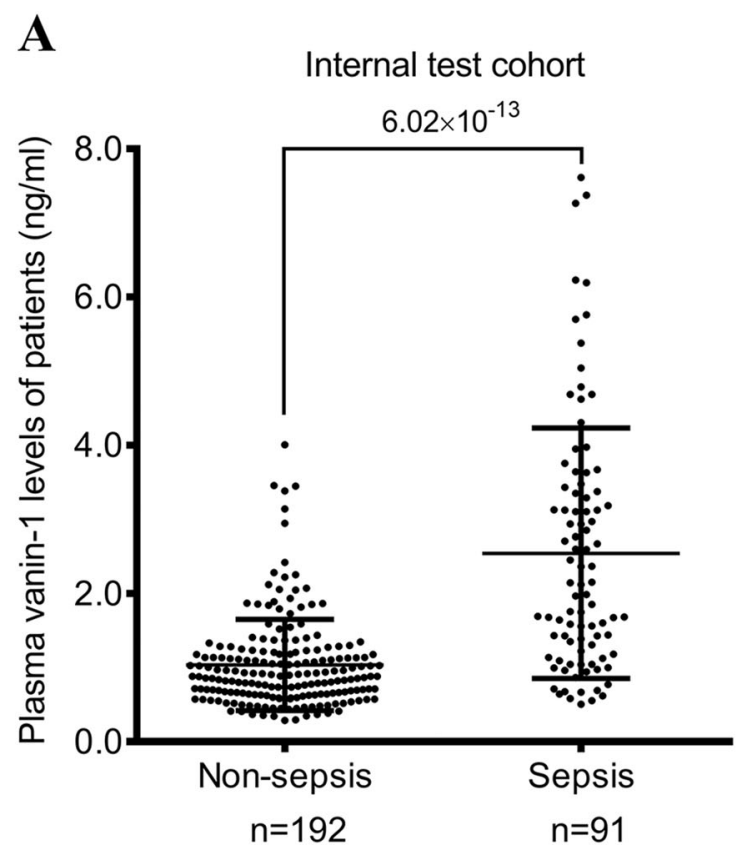

Fig. 2 Plasma vanin-1 in trauma patients with and without sepsis for the $\mathbf{a}$ internal test and $\mathbf{b}$ external validation cohorts. Patients who developed sepsis had significantly higher levels of plasma vanin-1 when
B

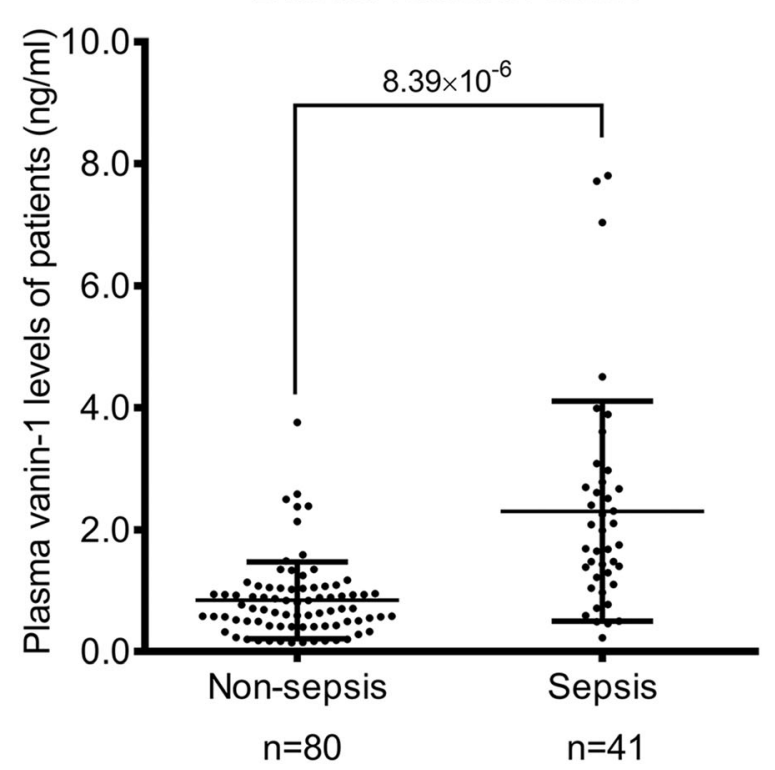

compared with patients who did not develop sepsis at day 1 after trauma. All samples were collected on day 1 after injury. Black bars show the mean and 95\% CI. Statistical analysis comprised Student's $t$ test

Table 2 Associations between each biomarker and traumatic sepsis in adjusted logistic regression models

\begin{tabular}{|c|c|c|c|c|}
\hline \multirow[t]{2}{*}{ Variables } & \multicolumn{2}{|c|}{ Internal test cohort } & \multicolumn{2}{|c|}{ External validation cohort } \\
\hline & $\overline{\text { OR }(95 \% \mathrm{CI})}$ & $P^{*}$ & $\overline{\mathrm{OR}}(\mathbf{9 5 \%} \mathrm{CI})$ & $P^{*}$ \\
\hline APACHE II & $1.17(1.10-1.23)$ & $5.35 \times 10^{-8}$ & $1.21(1.08-1.37)$ & $1.00 \times 10^{-3}$ \\
\hline PCT & $1.06(1.02-1.10)$ & $2.00 \times 10^{-3}$ & $0.99(0.90-1.08)$ & 0.86 \\
\hline CRP & $1.01(1.00-1.01)$ & $3.00 \times 10^{-3}$ & $1.01(1.00-1.02)$ & 0.05 \\
\hline Vanin-1 & $3.92(2.68-5.72)$ & $1.62 \times 10^{-12}$ & $4.26(2.22-8.17)$ & $1.28 \times 10^{-5}$ \\
\hline
\end{tabular}

APACHE II Acute Physiology and Chronic Health Evaluation II, PCT procalcitonin, CRP C-reactive protein *Adjusted for age, sex, smoking, drinking, and ISS

$70.73 \%$ and $90.00 \%$, respectively (Table S1 in the electronic supplementary material for details). We also analyzed the associations between CRP, PCT, APACHE II, and traumatic sepsis. Except for the PCT, the remaining CRP and APACHE II were related to the risk of sepsis after trauma (Table 2 and Table S2 in the electronic supplementary material). When compared with CRP $\quad($ AUC $=0.59$, 95\% CI 0.500-0.68, $\left.P=1.20 \times 10^{-3}\right), \quad$ PCT $(\mathrm{AUC}=0.63, \quad 95 \%$ CI $0.54-0.71, \quad P=1.30 \times$ $\left.10^{-3}\right)$ and APACHE II (AUC $=0.72$, 95\% CI 0.63-0.80, $P=0.09$ ), vanin-1 also afforded a better predictive ability (Fig. $3 \mathrm{~b}$ and Table 3). Furthermore, combining plasma 


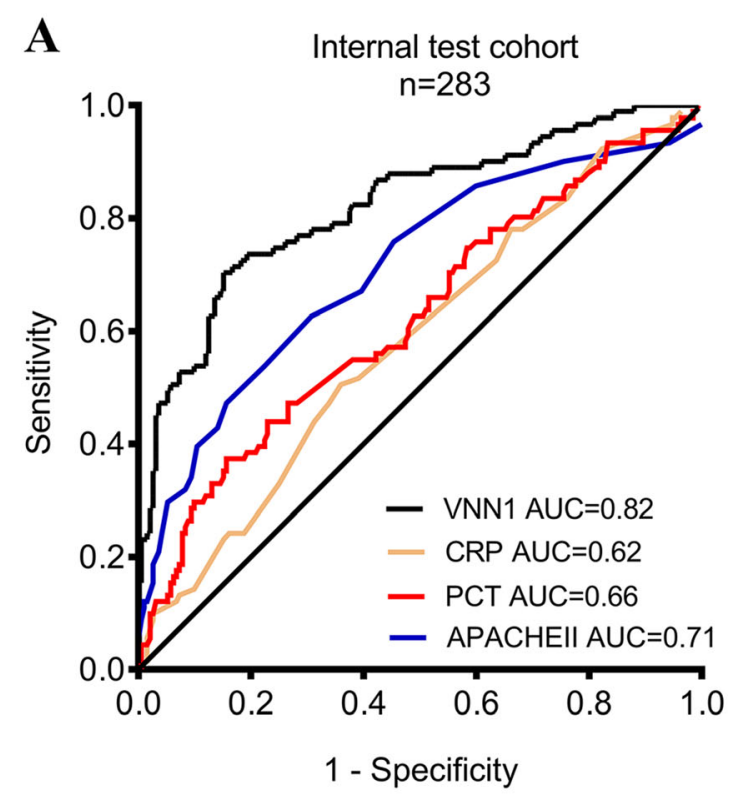

Fig. 3 Receiver operating curve (ROC) analysis of VNN1, CRP, PCT, and APACHE II for sepsis after trauma. Plasma vanin-1 afforded the best predictive value

vanin-1 with APACHE II increased the diagnostic efficiency $(\mathrm{AUC}=0.85$, Table 3$)$.

\section{DISCUSSION}

Increasing evidence has highlighted the importance of vanin-1 in various cancer and inflammatory disorders, including septic shock [17]. However, whether vanin-1 could be used as a biomarker for early warning of traumatic sepsis was still unknown. In this study, elevated admission plasma vanin-1 levels were observed in severe trauma patients. We have demonstrated that increased admission vanin-1 was significantly associated with the incidence of sepsis in severe trauma patients. Furthermore, after adjustment for age, sex, smoking, drinking, and ISS, vanin-1 was strongly associated with sepsis. When plasma vanin-1 was used as a biomarker for the early prediction of traumatic sepsis, better predictive abilities were obtained than PCT, CRP, and APACHE II in both of our internal test and external validation cohorts.

In previous studies, VNN1 mRNA had been used to predict the risk or prognosis of patients
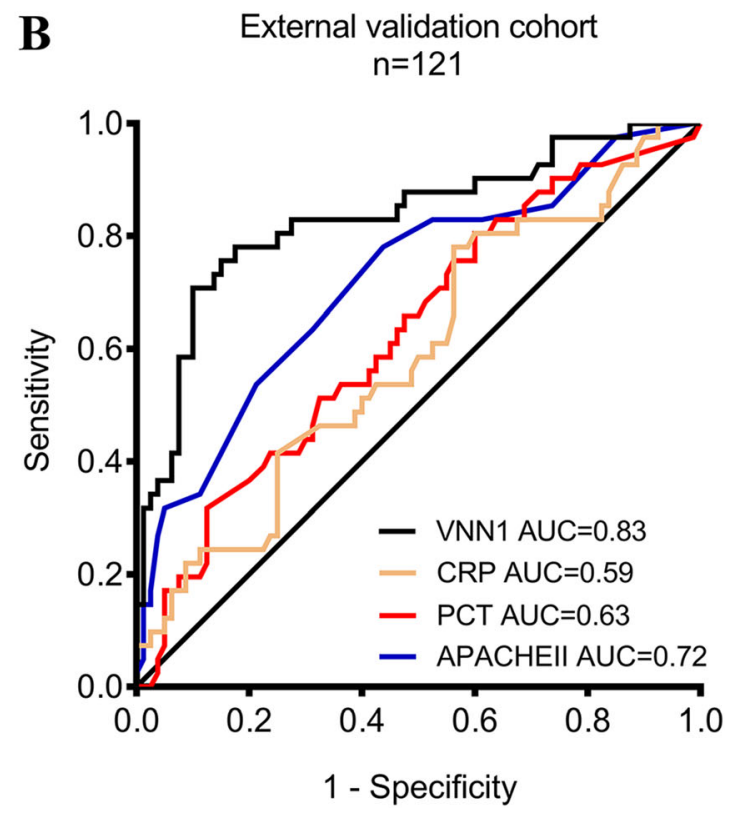

compared to other biomarkers and scores in the internal test cohort $(\mathbf{a}, n=283)$ and external validation cohort $(\mathbf{b}$, $n=121)$

with colorectal cancer $[18,19]$ and patients with acute myeloid leukemia [20]. Elevated circulation or urinary vanin-1 has been reported in acute/chronic kidney injury [21, 22], drug-induced renal injury [23], and patients with asthma [24]. We demonstrated in the current study that plasma vanin-1 increased rapidly after injury. Specially, patients who developed sepsis later had higher plasma vanin-1 on admission. It was consistent with the findings from our group through reanalyzing genomewide expression of leukocytes from trauma patients in a public trauma website (The Human Genomic Response to Severe Traumatic Injury, http://web.mgh.harvard.edu/TRT/). In this public database, VNN1 mRNA was upregulated and differentially expressed between the complicated recovery group and the uncomplicated recovery group within $12 \mathrm{~h}$ and at $1,4,7,14$, 21 , and 28 days after injury. We further analyzed the public transcriptome data of patients with sepsis from the GEO dataset (https://www. ncbi.nlm.nih.gov/gds/). Upregulation of VNN1 mRNA in patients with sepsis was obviously detected in the GSE28750 [25], GSE64457 [26], 


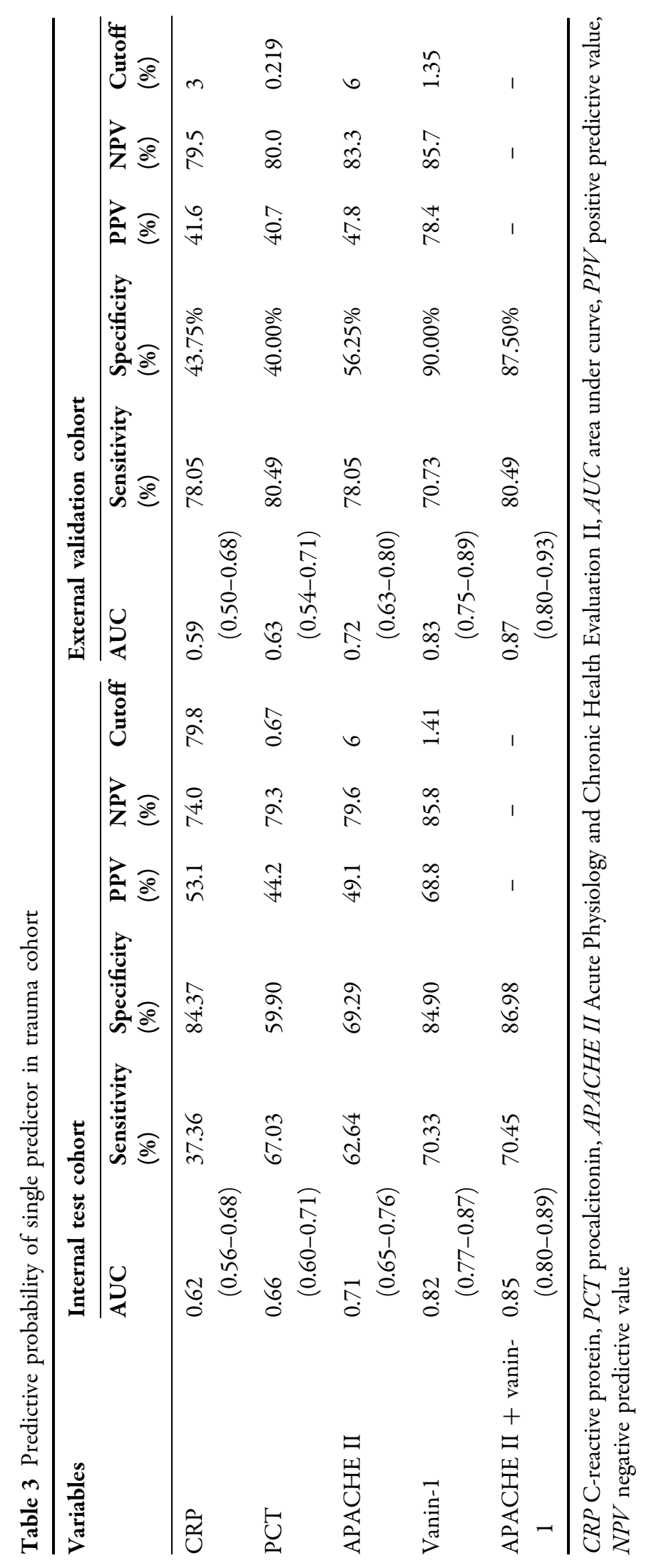


and GSE46995 [27]. It has been controversial whether PCT and CRP could be used to predict the incidence of sepsis in trauma patients [28]. In this study, we showed that both PCT and CRP were not suitable for diagnosing sepsis in severe trauma patients, and have low sensitivity or specificity. In our two larger trauma cohorts, plasma vanin- 1 at day 1 after trauma was independently associated with the incidence of traumatic sepsis even after adjustment for age, sex, smoking, drinking, and ISS in the multiple logistic regression analysis. Since the internal test cohort and external validation cohort were enrolled from two different hospitals, we considered it was more reasonable to reanalyze the cutoff. Fortunately, the cutoff value in the internal test cohort was consistent with that of the external validation cohort $(1.41 \mathrm{ng} / \mathrm{ml}$ vs. $1.35 \mathrm{ng} / \mathrm{ml}$ ). Our results showed that plasma vanin-1 level on admission could be used to predict the risk of sepsis after trauma with an AUC over 0.80. Compared with CRP, PCT, and APACHE II, plasma vanin-1 outperformed the predicted ability in our study cohorts. When vanin-1 was combined with the APACHE II score, the AUC could be increased to 0.85. All of these findings supported increased vanin-1 in trauma patients, and plasma vanin-1 might be used as a potential biomarker of post-injury sepsis at an early stage. The potential relevance of plasma vanin-1 would help clinicians categorize high-risk patients with sepsis at the early stage of trauma and enable rapid treatment, improve outcomes, and reduce unnecessary antibiotic therapy.

To our knowledge, little has been known about the mechanisms of how vanin-1 influences the process of sepsis. VNN1 might play a protective role against oxidative stress and the inflammatory response caused by infection $[29,30]$. Yamashita et al. reported vanin-1 elevation in patients with influenza A (H1N1) pneumonia [31]. Furthermore, VNN1 mRNA increased significantly in the human alveolar epithelial carcinoma cell line A549 in response to tumor necrosis factor alpha (TNF $\alpha$ ), interleukin-6 (IL-6), IL-1 $\beta$, lipopolysaccharides (LPS), and $\mathrm{H}_{2} \mathrm{O}_{2}$. These studies indicated that the strong oxidative stress caused by injury also induced the upregulation of VNN1 mRNA. In addition, injury and subsequent pro-inflammatory cytokines, such as TNF $\alpha$ and IL-1 $\beta$, might upregulate VNN1 mRNA levels [31, 32]. Additionally, previous studies demonstrated that glutathione, the most potent cellular antioxidant, was elevated in $V N N 1^{-/-}$mice, resulting in a lack of cysteamine in tissues. Therefore, $V N N 1^{-1-}$ mice exhibited resistance to oxidative damage and reduced inflammatory responses [11]. Moreover, there was a negative correlation between the expression levels of VNN1 and peroxisome proliferator-activated receptor- $\gamma$ $(\operatorname{PPAR} \gamma)$ [33]. PPAR $\gamma$ in monocytes plays an antiinflammatory role by inhibiting other transcription factors, such as nuclear factor- $\kappa \mathrm{B}$ and activator protein $1[34,35]$. VNN1 promotes inflammation partly by inhibiting both PPAR $\gamma$ expression and signal transduction [32, 33]. Ling et al. suggested that VNN1 was negatively regulated by miR-203 and affected sepsis through the AKT signaling pathway. Therefore, we speculated that $V N N 1$ played an important role in sepsis development by regulating the glutathione, cysteamine, PPAR $\gamma$, and AKT signaling pathways. Further functional experiments might help us to clarify the role of $V N N 1$ in sepsis development.

Some potential limitations of the present study should be considered. First, the sample size of patients in each cohort was relatively small, notably in the discovery cohort. Additional large studies with multiple ethnicities are needed to validate these findings. Second, we revealed that plasma vanin-1 levels were associated with traumatic sepsis. However, how vanin-1 affected the development of sepsis was unclear. More studies with cellular and molecular experiments were required to investigate the relevant mechanisms. Finally, although the correlations between plasma vanin-1 and traumatic sepsis were validated, whether the findings could be interpreted in other ethnic groups needs further evaluation.

\section{CONCLUSION}

Our study demonstrated that plasma vanin-1 on admission is independently associated with the risk of traumatic sepsis and has a good capacity 
to predict sepsis at the early stage of trauma. To confirm these findings, further studies with multiple populations and functional evaluations are warranted.

\section{ACKNOWLEDGEMENTS}

We acknowledge Dr. Dongpo Jiang and Lianyang Zhang, Army Medical University, for the collection of blood samples and clinical information. Hongxiang $\mathrm{Lu}$ and Anqiang Zhang contributed equally to this work. We thank all the participants who participated in this study.

Funding. This work is supported by Science and Technology Innovation Project for Academicians of Chongqing (cstc2019jcyjmsxmX0266), National Natural Science Foundation of China (81772061, 81971830), Medical Research Funding of PLA of China (AWS14C003). The Rapid Service Fee was funded by the authors.

Authorship. All named authors meet the International Committee of Medical Journal Editors (ICMJE) criteria for authorship for this article, take responsibility for the integrity of the work as a whole, and have given their approval for this version to be published.

Authorship Contributions. WG, AZ and JJ designed the study. HL and $A Z$ were responsible for data collection and management. DW, JD, DD and JS were responsible for sample collection and laboratory processing. HL and LQ performed statistical analysis and drafted the manuscript. WG, AZ and JJ critically revised the manuscript. All authors read and approved the final manuscript.

Disclosures. Hongxiang Lu, Anqiang Zhang, Dalin Wen, Juan Du, Jianhui Sun, Liang Qiao, Dingyuan Du, Wei Gu and Jianxin Jiang have nothing to disclose.

Compliance with Ethics Guidelines. The research was approved by the Institutional Ethics Review Board of the Army Medical University (reference number [2012]009). The study was performed in accordance with the Helsinki Declaration of 1964, and its later amendments. Informed consent of all patients was obtained from the patients or their relatives.

Data Availability. The datasets used and analyzed during the current study are available from the corresponding author in response to reasonable requests.

Open Access. This article is licensed under a Creative Commons Attribution-NonCommercial 4.0 International License, which permits any non-commercial use, sharing, adaptation, distribution and reproduction in any medium or format, as long as you give appropriate credit to the original author(s) and the source, provide a link to the Creative Commons licence, and indicate if changes were made. The images or other third party material in this article are included in the article's Creative Commons licence, unless indicated otherwise in a credit line to the material. If material is not included in the article's Creative Commons licence and your intended use is not permitted by statutory regulation or exceeds the permitted use, you will need to obtain permission directly from the copyright holder. To view a copy of this licence, visit http://creativecommons.org/licenses/by$\mathrm{nc} / 4.0 /$.

\section{REFERENCES}

1. Bohmer $\mathrm{AB}$, Just $\mathrm{KS}$, Lefering $\mathrm{R}$, et al. Factors influencing lengths of stay in the intensive care unit for surviving trauma patients: a retrospective analysis of 30,157 cases. Crit Care. 2014;18(4):R143.

2. Zhang AQ, Gu W, Zeng L, et al. Genetic variants of microRNA sequences and susceptibility to sepsis in patients with major blunt trauma. Ann Surg. 2015;261(1):189-96.

3. Lamparello AJ, Namas RA, Constantine G, et al. A conceptual time window-based model for the early stratification of trauma patients. J Intern Med. 2019;286(1):2-15.

4. Haagsma JA, Graetz N, Bolliger I, et al. The global burden of injury: incidence, mortality, disability- 
adjusted life years and time trends from the Global Burden of Disease study 2013. Inj Prev. 2016;22(1): 3-18.

5. Vourc'h M, Roquilly A, Asehnoune K. Trauma-induced damage-associated molecular patterns-mediated remote organ injury and immunosuppression in the acutely Ill patient. Front Immunol. 2018;9: 1330 .

6. Xiao W, Mindrinos MN, Seok J, et al. A genomic storm in critically injured humans. J Exp Med. 2011;208(13):2581-90.

7. Lindner HA, Balaban U, Sturm T, Weiss C, Thiel M, Schneider-Lindner V. An algorithm for systemic inflammatory response syndrome criteria-based prediction of sepsis in a polytrauma cohort. Crit Care Med. 2016;44(12):2199-207.

8. Asehnoune K, Balogh Z, Citerio G, et al. The research agenda for trauma critical care. Intensive Care Med. 2017;43(9):1340-51.

9. Naquet P, Pitari G, Dupre S, Galland F. Role of the Vnn1 pantetheinase in tissue tolerance to stress. Biochem Soc Trans. 2014;42(4):1094-100.

10. Nitto T, Onodera K. Linkage between coenzyme a metabolism and inflammation: roles of pantetheinase. J Pharmacol Sci. 2013;123(1):1-8.

11. Berruyer C, Martin FM, Castellano R, et al. Vanin1-/- mice exhibit a glutathione-mediated tissue resistance to oxidative stress. Mol Cell Biol. 2004;24(16):7214-24.

12. Martin F, Penet MF, Malergue F, et al. Vanin-1(-/-) mice show decreased NSAID- and Schistosoma-induced intestinal inflammation associated with higher glutathione stores. J Clin Invest. 2004;113(4):591-7.

13. Meghari S, Berruyer C, Lepidi H, Galland F, Naquet $\mathrm{P}$, Mege JL. Vanin-1 controls granuloma formation and macrophage polarization in Coxiella burnetii infection. Eur J Immunol. 2007;37(1):24-32.

14. Sandesc M, Rogobete AF, Bedreag OH, et al. Analysis of oxidative stress-related markers in critically ill polytrauma patients: an observational prospective single-center study. Bosn J Basic Med Sci. 2018;18(2):191-7.

15. Singer M, Deutschman CS, Seymour CW, et al. The third international consensus definitions for sepsis and septic shock (Sepsis-3). JAMA. 2016;315(8): 801-10.

16. Chung KP, Chen GY, Chuang TY, et al. Increased plasma acetylcarnitine in sepsis is associated with multiple organ dysfunction and mortality: a multicenter cohort study. Crit Care Med. $2019 ; 47(2): 210-8$.

17. Ling L, Lu HT, Wang HF, Shen MJ, Zhang HB. MicroRNA-203 acts as a potent suppressor in septic shock by alleviating lung injury via inhibition of VNN1. Kidney Blood Press Res. 2019;44(4):565-82.

18. Yip KT, Das PK, Suria D, Lim CR, Ng GH, Liew CC. A case-controlled validation study of a blood-based seven-gene biomarker panel for colorectal cancer in Malaysia. J Exp Clin Cancer Res. 2010;29:128.

19. Marshall KW, Mohr S, Khettabi FE, et al. A bloodbased biomarker panel for stratifying current risk for colorectal cancer. Int J Cancer. 2010;126(5): 1177-86.

20. Izzi V, Lakkala J, Devarajan R, et al. Vanin 1 (VNN1) levels predict poor outcome in acute myeloid leukemia. Am J Hematol. 2018;93(1):E4-7.

21. Washino S, Hosohata K, Oshima M, et al. A novel biomarker for acute kidney injury, vanin-1, for obstructive nephropathy: a prospective cohort pilot study. Int J Mol Sci. 2019;20(4):899.

22. Hosohata K, Matsuoka H, Iwanaga K, Kumagai E. Urinary vanin-1 associated with chronic kidney disease in hypertensive patients: a pilot study. J Clin Hypertens (Greenwich). 2020;22(8):1458-65.

23. Hosohata $\mathrm{K}$, Ando $\mathrm{H}$, Fujiwara $\mathrm{Y}$, Fujimura A. Vanin-1: a potential biomarker for nephrotoxicantinduced renal injury. Toxicology. 2011;290(1): 82-8.

24. Xiao C, Biagini Myers JM, Ji H, et al. Vanin-1 expression and methylation discriminate pediatric asthma corticosteroid treatment response. J Allergy Clin Immunol. 2015;136(4):923-31 e3.

25. Sutherland A, Thomas M, Brandon RA, et al. Development and validation of a novel molecular biomarker diagnostic test for the early detection of sepsis. Crit Care. 2011;15(3):R149.

26. Demaret J, Venet F, Friggeri A, et al. Marked alterations of neutrophil functions during sepsis-induced immunosuppression. J Leukoc Biol. 2015;98(6):1081-90.

27. Shalova IN, Lim JY, Chittezhath M, et al. Human monocytes undergo functional re-programming during sepsis mediated by hypoxia-inducible factor1alpha. Immunity. 2015;42(3):484-98.

28. Ciriello V, Gudipati S, Stavrou PZ, Kanakaris NK, Bellamy MC, Giannoudis PV. Biomarkers predicting sepsis in polytrauma patients: current evidence. Injury. 2013;44(12):1680-92. 
29. Berruyer C, Pouyet L, Millet V, et al. Vanin-1 licenses inflammatory mediator production by gut epithelial cells and controls colitis by antagonizing peroxisome proliferator-activated receptor gamma activity. J Exp Med. 2006;203(13):2817-27.

30. Pouyet L, Roisin-Bouffay C, Clement A, et al. Epithelial vanin-1 controls inflammation-driven carcinogenesis in the colitis-associated colon cancer model. Inflamm Bowel Dis. 2010;16(1):96-104.

31. Yamashita N, Yashiro M, Ogawa H, et al. Metabolic pathway catalyzed by vanin-1 pantetheinase plays a suppressive role in influenza virus replication in human alveolar epithelial A549 cells. Biochem Biophys Res Commun. 2017;489(4):466-71.

32. Jansen PA, Kamsteeg M, Rodijk-Olthuis D, et al. Expression of the vanin gene family in normal and inflamed human skin: induction by proinflammatory cytokines. J Invest Dermatol. 2009;129(9): 2167-74.

33. Zhang B, Lo C, Shen L, et al. The role of vanin-1 and oxidative stress-related pathways in distinguishing acute and chronic pediatric ITP. Blood. 2011;117(17):4569-79.

34. Ricote M, Glass CK. PPARs and molecular mechanisms of transrepression. Biochim Biophys Acta. 2007;1771(8):926-35.

35. Szeles L, Torocsik D, Nagy L. PPARgamma in immunity and inflammation: cell types and diseases. Biochim Biophys Acta. 2007;1771(8): 1014-30. 